\title{
Measuring absolute number concentrations of nanoparticles using single-particle tracking
}

\author{
Magnus Röding, , $^{, *}$ Hendrik Deschout, ${ }^{2,3}$ Kevin Braeckmans, ${ }^{2,3}$ and Mats Rudemo ${ }^{1}$ \\ ${ }^{1}$ Department of Mathematical Statistics, Chalmers University of Technology and Gothenburg University, Gothenburg, Sweden \\ ${ }^{2}$ Laboratory of General Biochemistry and Physical Pharmacy, Ghent University, Ghent, Belgium \\ ${ }^{3}$ Center for Nano- and Biophotonics, Ghent University, Ghent, Belgium
}

(Received 21 February 2011; revised manuscript received 4 July 2011; published 20 September 2011)

\begin{abstract}
Single-particle tracking (SPT) microscopy is increasingly used to characterize nanoparticulate systems. We introduce a concept for estimation of particle number concentration in Brownian particle dispersions using SPT based on a model for the trajectory length distribution of particles to estimate the detection region volume. The resulting method is independent of precalibration reference measurements, and robust with respect to image processing settings. Experimentally estimated concentrations of different dilutions of 0.19 - and $0.52-\mu \mathrm{m}$ polymer nanospheres are in excellent agreement with estimates computed from the concentrations of the stock solutions.

DOI: 10.1103/PhysRevE.84.031920

PACS number(s): 87.64.M-, 87.15.Vv, 87.64.kv, 87.85.Qr
\end{abstract}

\section{INTRODUCTION}

In the last decade, there has been a rapid growth in the design of functional nanoparticles. For instance, nanomedicines are being developed to deliver therapeutic agents to specific target tissues [1]. In biomedical imaging, gold nanoparticles are used for x-ray computer tomography, iron oxide particles for NMR imaging, and quantum dots for fluorescence imaging [2]. Apart from manmade nanoparticles, there is also a growing interest in detecting and characterizing sub- $\mu \mathrm{m}$ aggregates for biomedical diagnostics. One example is the detection of blood-borne specific cell-derived microparticles, which could serve as biomarkers for metabolic and systemic diseases, as well as for thrombosis and cancer $[3,4]$. Another example is the detection of amyloid $\beta$ aggregates in cerebrospinal fluid or plasma, which could allow early diagnosis and prognosis of neurodegenerative disorders such as Alzheimer's [5].

There is currently an active search for methods that can accurately detect and characterize nanoparticulate matter [6-8], especially in blood, tissues, or cells [9-12]. We recently demonstrated that (fluorescence) single-particle tracking (SPT) is a powerful technique that can accurately measure the size distribution of (fluorescently labeled) nanoparticles in undiluted complex biological fluids like blood [11]. By imaging the diffusive motion of (fluorescently labeled) nanoparticles, the diffusion coefficient and size can be readily obtained for each individual particle, resulting in accurate size distribution estimates. In addition, qualitative information on the particle number concentration is available since the number of detected particles or trajectories is directly proportional to their concentration. Thus SPT is a powerful technique for the unperturbed characterization of size and concentration of nanoparticles in a dispersion.

However, to measure the absolute number concentration, the volume of the three-dimensional (3D) detection region must be accurately known. One way of doing this is to perform a precalibration using reference particles, as was recently suggested [13]. While this is a useful first approximation, it should be realized that the detection region depends on several variable factors. Indeed, apart from the optical system,

*Corresponding author: roding@ chalmers.se the detection region is also affected by particle brightness and image processing settings used for particle detection and calculation of the motion trajectories.

In this work, we present a framework to estimate the detection region volume from the SPT data themselves, hence eliminating the need to perform separate calibration measurements. The estimation of the detection region volume is performed by estimation of the axial size of the detection region. This is related to the probability distribution of trajectory lengths within the detection region, which can be obtained numerically as a function of the axial size. By use of simulations and experimental data we demonstrate that accurate, absolute nanoparticle concentrations can be measured over a wide range and that the method is robust with respect to image processing settings.

\section{THEORY}

Consider a tracking experiment where particles are diffusing freely in a liquid suspension. The concentration is sufficiently low to make particle interaction negligible. A Brownian motion characterized by the usual mean squared deviation property [14] describes the diffusive motion in $\mathcal{N}$ dimensions, where $\mathcal{N}=3$ for particles moving in space. If a particle is observed at $K$ equidistant time points ( $K$ consecutive frames of a time-lapse video sequence) with sampling interval $\Delta t$, the $K$ positions denoted by $r_{1}, r_{2}, \ldots, r_{K}$ can be written as the previous position plus a Gaussian increment, $r_{i}=r_{i-1}+\Delta G$, where $\Delta G$ is an $\mathcal{N}$-dimensional vector of normally distributed independent components, each with mean zero and variance $2 D \Delta t$. We refer to $K$ as the trajectory length. The trajectory length depends primarily on the size of the detection region and the diffusion coefficient.

The detection region is modeled as a rectangular box (see Fig. 1). The lateral sizes are determined by the microscope field of view. The axial size, which we will refer to as the tracking depth, depends on the depth of field of the microscope and the parameters of the particle detection algorithm, as well as of the brightness of the observed particles. Since the latter two properties are not fixed but can vary strongly between experiments, the effective thickness of the detection region can vary as well, due to particles becoming gradually less 


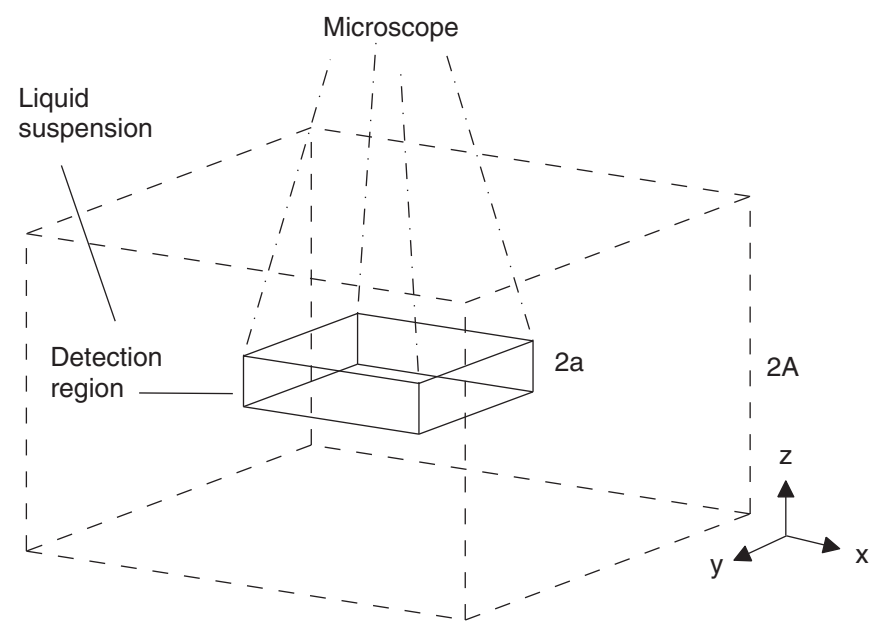

FIG. 1. An illustration of the experimental setup. The detection region is modeled as a rectangular box centered in the liquid suspension. Particles outside the detection region cannot be observed. The tracking depth is $2 a$, and the thickness of the liquid suspension is $2 A$.

bright and more diffuse when moving away from the focal plane. Therefore particles will not be detected anymore by the image processing software from a certain out-of-focus distance depending on the particle brightness and image processing settings (see Appendix C). Note that the field of view (i.e., the lateral sizes of the detection region) can be calibrated separately (e.g., through a calibration grid), so that it is only the tracking depth that is unknown. Since the lateral sizes of the detection region are typically much larger than the axial size, a simplification will be introduced by assuming that particles are entering and exiting the detection region only by means of axial diffusion, i.e., parallel to the optical axis ( $z$ direction). The three-dimensional (3D) system is hence replaced by a one-dimensional (1D) model.

\section{A. Trajectory length distribution}

Assume that particles are diffusing within the region $[-A, A]$. They are visible within the detection region $[-a, a]$, and invisible otherwise. Hence the thickness of the liquid suspension is $2 A$ and the tracking depth is $2 a$. We assume here that the distance from the detection region to the nearest boundary of the suspension is much larger than the characteristic magnitude of a spatial increment of a particle (within the time interval $\Delta t$ ). Furthermore, assuming diffusion equilibrium, particles are uniformly distributed over $[-A, A]$. Consequently, particles outside of the detection region are uniformly distributed over $[-A,-a] \cup[a, A]$. The position of these particles in the following time step will be their current position plus a Gaussian increment. The probability density of the particle positions in the following time step is hence a convolution of a uniform density with a Gaussian density. Restricting to the interval $[-a, a]$ and normalizing, we obtain the conditional probability density $f(z)$ of the position of a particle that has just entered the detection region,

$$
f(z)= \begin{cases}\frac{h(z)}{\int_{-a}^{a} h(z) d z}, & z \in[-a, a], \\ 0, & z \notin[-a, a]\end{cases}
$$

where, letting $\phi$ denote the standard normal density and $\Phi$ the standard normal cumulative distribution function,

$$
\begin{aligned}
h(z)= & \frac{1}{2(A-a)}\left[\Phi\left(\frac{z+A}{\sqrt{2 D \Delta t}}\right)-\Phi\left(\frac{z+a}{\sqrt{2 D \Delta t}}\right)\right. \\
& \left.+\Phi\left(\frac{z-a}{\sqrt{2 D \Delta t}}\right)-\Phi\left(\frac{z-A}{\sqrt{2 D \Delta t}}\right)\right]
\end{aligned}
$$

is the non-normalized density of particles just having entered the detection region (see Appendix A). We want to compute the probability distribution of the trajectory length once a particle enters the detection region. We define $f_{k}$ as the (nonnormalized) density of the position of a particle after $k$ steps assuming that $K \geqslant k$, more precisely $f_{k}(z)=d / d z\left[P\left(Z_{k} \leqslant\right.\right.$ $z$ and $K \geqslant k)$ ], for $k \geqslant 1$. By definition $f_{k}(z)$ is zero outside of $[-a, a]$. For the first position that the particle is within the detection region, i.e., $k=1$, the probability density is given by Eq. (1). To compute the probability density of the particle after step 2, $f_{1}$ is convolved with the Gaussian propagator

$$
G(z)=\frac{1}{\sqrt{2 D \Delta t}} \phi\left(\frac{z}{\sqrt{2 D \Delta t}}\right) .
$$

However, since we have assumed that the particle resides in the detection region for $K$ steps ( $K$ now assumed larger than 1), it cannot be outside $[-a, a]$ and the probability density has to be truncated. More generally, the density $f_{k}$ can be calculated from $f_{k-1}$ according to

$$
f_{k}(z)= \begin{cases}\int_{-\infty}^{\infty} f_{k-1}\left(z_{0}\right) G\left(z-z_{0}\right) d z_{0}, & z \in[-a, a], \\ 0, & z \notin[-a, a]\end{cases}
$$

for $k>1$, where the convolution with the Gaussian propagator represents the diffusive spread of the particles and the truncation represents that particles exiting the detection region once are not part of the same trajectory. Thus we can compute $f_{k}$ recursively from Eq. (4) using $f_{1}=f$ as given by Eq. (1). The probability that the particle resides in the detection region for at most $k$ consecutive frames is

$$
P_{a}(K \leqslant k)=1-\int_{-a}^{a} f_{k+1}(z) d z
$$

where the dependence on $a$ is emphasized. Computing $f_{k}$ for $k \geqslant 1$ cannot be done analytically. We use a fast numerical scheme, where the densities are expanded (approximated) in a space of equidistant translates of a Gaussian kernel, reducing the convolutions to a set of matrix operations identical in every time step [15]. The probability distribution for the trajectory length $K$ is then

$$
P_{a}(K=k)=P_{a}(K \leqslant k)-P_{a}(K \leqslant k-1),
$$

which is implicitly a function of $a$ and $D$. The numerical convolution scheme is described in detail in Appendix B. We emphasize here that the computations for the trajectory length distribution are performed in discrete time. Hence the usual approaches for solving the heat equation (in continuous time) using series expansions will not be useful to us, since not taking the discrete nature of the tracking into account would render incorrect trajectory length distributions.

Since an empirical distribution of trajectory lengths is readily available from an SPT experiment, we can now use our 
theoretical model to estimate the axial extent $a$ of the detection region. Suppose that we have a monodisperse ensemble of particles in a liquid suspension with known diffusion coefficient. The latter is a reasonable assumption since the diffusion coefficient is readily estimated from the particle trajectories [16]. We can calculate a maximum likelihood estimate $\hat{a}$ of $a$ by fitting the trajectory length distribution according to Eq. (6) to an experimental trajectory length distribution. Suppose we have observed trajectories each with a length $K \geqslant k_{\min }$ (it is typical to impose a lower threshold like $K \geqslant 3$ or $K \geqslant 4$ for the trajectory length, since short trajectories are more likely to be false positives $[11,17])$, and let the number of observed trajectories of length $k$ be $N_{k}$. The log-likelihood function is

$$
l(a)=\sum_{k \geqslant k_{\min }} N_{k} \ln P_{a}\left(K=k \mid K \geqslant k_{\min }\right),
$$

where

$$
P_{a}\left(K=k \mid K \geqslant k_{\min }\right)=\frac{P_{a}(K=k)}{P_{a}\left(K \geqslant k_{\min }\right)},
$$

where $P_{a}\left(K \geqslant k_{\min }\right)$ is given as $1-P_{a}\left(K \leqslant k_{\min }-1\right)$ in terms of Eq. (5). The maximum likelihood estimate $\hat{a}$ is the value of $a$ that maximizes $l(a)$ [18].

\section{B. Number concentration}

Having estimated the tracking depth, it is now possible to estimate the number concentration. Let $\bar{N}$ denote the mean number of particles per video frame. The point estimate for the number concentration is

$$
\hat{c}=\frac{\bar{N}}{8 \hat{a} a_{x} a_{y} 10^{-12}} \text { particles } / \mathrm{ml},
$$

where $2 a_{x}$ and $2 a_{y}$ are the lateral sizes in $\mu \mathrm{m}$ of the detection region. We can estimate $\bar{N}$ by counting the number of trajectories as follows. Let $n$ be the number of frames. Let $N_{k}$ as earlier be the number of observed trajectories of length $k$. The number of observed particle positions is the sum of all the trajectory lengths. Dividing by the number of frames, this provides an estimate of the mean number of particles per frame. We estimate $\bar{N}$ by

$$
\bar{N}=\frac{1}{\hat{p}_{\text {obs }}} \frac{1}{n} \sum_{k \geqslant k_{\min }} k N_{k} .
$$

The factor $\hat{p}_{\text {obs }}$ is introduced to correct for the underestimation of the concentration, which results from discarding trajectories with length $k<k_{\min }$. Specifically, $\hat{p}_{\text {obs }}$ is the estimated probability of a random particle position within the detection region to be observed, i.e., part of a trajectory with $k \geqslant k_{\min }$,

$$
\hat{p}_{\text {obs }}=\frac{\sum_{k \geqslant k_{\min }} k P_{\hat{a}}(K=k)}{\sum_{k \geqslant 1} k P_{\hat{a}}(K=k)} .
$$

Using this correction factor we can make $\bar{N}$ approximately unbiased. The standard error of the concentration estimate can also be assessed using bootstrapping [19]. It is natural to perform the bootstrapping on the "video level," since the videos are (approximately) independent. We extract $\mathcal{B}$ bootstrap samples by sampling from the set of videos, with replacement, $\mathcal{B}$ times. We compute $\mathcal{B}$ estimates of the concentration, $\hat{c}_{1}, \ldots, \hat{c}_{\mathcal{B}}$, and compute an approximate standard deviation of the estimate by

$$
\sigma_{\hat{c}}=\sqrt{\frac{1}{\mathcal{B}-1} \sum_{i=1}^{\mathcal{B}}\left(\hat{c}_{i}-c_{\text {mean }}\right)^{2}},
$$

where $c_{\text {mean }}$ is just the average of the bootstrap estimates. Note that this method relies on all videos being (approximately) equally long. The simulation study described below leads to the conclusion that the tracking depth estimate and the concentration estimate are approximately unbiased, and that the bootstrap standard errors for $\mathcal{B}=50$ are very close to the actual standard errors.

\section{SIMULATION STUDY}

We performed a simulation study to validate the model and the estimates for the tracking depth and the concentration. We let noninteracting point particles diffuse in three dimensions in a virtual liquid suspension with periodic boundary conditions (hence the concentration is constant, with no net flux). A predetermined number of particles were initially placed randomly in the liquid suspension (all three coordinates being uniformly distributed). For each time increment $\Delta t$, particles performed random walk in each of the three directions, independent of the other directions, with normally distributed increments of mean zero and variance $2 D \Delta t$. When particles entered the virtual detection region, the trajectory length was recorded. The same particle was allowed to enter the detection region more than once. We let the lateral size of the detection region be $40 \mu \mathrm{m}$ (roughly similar to the parameters of the experimental data; see Appendix C) in both directions. We let the liquid suspension be $100 \mu \mathrm{m}$ in all three directions. The code was run in MATLAB.

We validated the estimate of the tracking depth parameter $a$ and the concentration on simulated data sets resembling the experimentally obtained data sets to be studied later on. We used three different diffusion coefficients, $D=1 \mu \mathrm{m}^{2} / \mathrm{s}$, $D=2 \mu \mathrm{m}^{2} / \mathrm{s}$, and $D=5 \mu \mathrm{m}^{2} / \mathrm{s}$ (corresponding roughly to the range $0.1-0.5 \mu \mathrm{m}$ in diameter for particles diffusing in water), and values of $a$ varying between 0.1 and 2 in increments of 0.1 . A total of 30 video sequences, each of length $10 \mathrm{~s}$ (250 frames, $\Delta t=40 \mathrm{~ms}$ ), were simulated in each run. The true concentration of particles was $c=10^{9}$ particles $/ \mathrm{ml}$ throughout (note that the concentration only affects for how long the system needs to be observed in order to acquire a sufficient amount of data). For both the tracking depth and the concentration, the mean estimates as computed from 20000 simulations for each data point (each combination of $a$ and $D$ ) are presented; see Fig. 2. We see that the bias increases (negatively for the tracking depth, positively for the concentration) as $a$ increases. It was found that this is not due to the finite size of the simulated data sets; this strongly suggests that the 1D approximation in the model for the trajectory length distribution is worse for larger values of $a$ relative to the lateral dimensions. However, while the use of the 1D approximative model clearly has limitations, the bias in the investigated range should be acceptable for practical use (for the experimental SPT measurements in this work, we can expect a bias of at most 5\%). Additionally, it was found (not shown) that using 50 or more bootstrap samples, the 

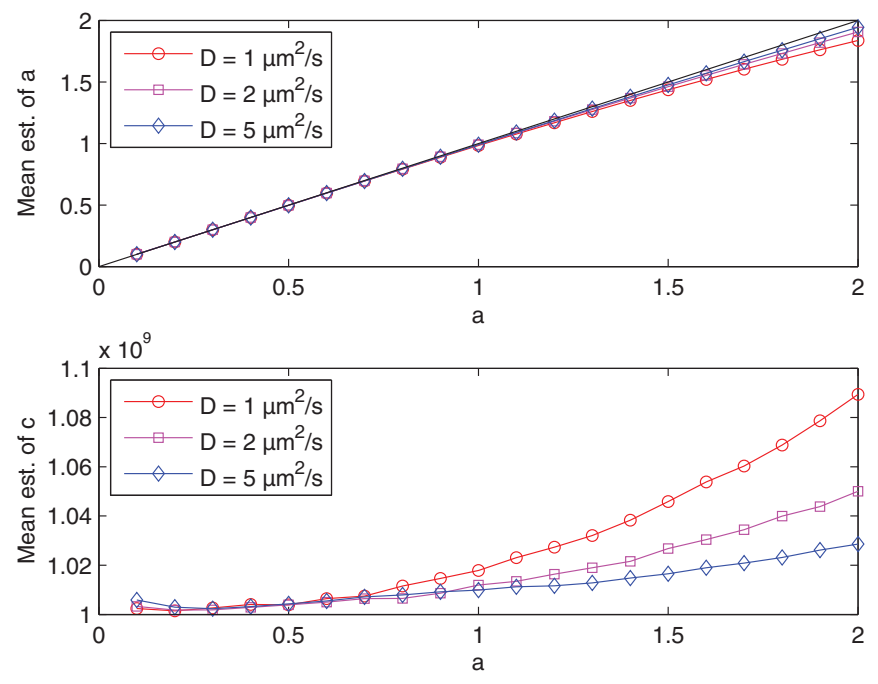

FIG. 2. (Color online) Simulation study of the tracking depth parameter estimate (upper) and the concentration estimate (lower). The mean estimates for $D=1 \mu \mathrm{m}^{2} / \mathrm{s}$ (red, circles), $D=2 \mu \mathrm{m}^{2} / \mathrm{s}$ (magenta, squares), and $D=5 \mu \mathrm{m}^{2} / \mathrm{s}$ (blue, diamonds) are shown as a function of the true value of $a$. The true values of $a$ are indicated with the black solid line. The true concentration of particles was $c=10^{9}$ particles $/ \mathrm{ml}$. The increasing bias (negative for $a$, positive for $c$ ) for increasing $a$ is due to the 1D approximation in the model for the trajectory length distribution.

standard error of both the tracking depth and the concentration estimates could be estimated quite precisely.

Sample size is an important issue. The precision of the estimates depends on the number of acquired videos, the length of the videos, the concentration, the tracking depth, and the diffusion coefficient (also these latter two affect the number of observed trajectories, i.e., the amount of data). It is important that each video is substantially longer than a typical trajectory length. Otherwise, the trajectory length distribution would be truncated and the estimations invalid. Determination of approximate data set sizes may be assessed by simulation. However, in practice the most straightforward method to find a suitable sample size is often to do sequential experiments, keeping in mind that to reduce the standard error by a factor of two and four times as many videos need to be acquired.

\section{EXPERIMENTAL RESULTS}

To experimentally verify the theory, SPT experiments were carried out on dispersions of fluorescent polymer nanospheres using a custom-built laser wide-field epifluorescence microscope setup. Using custom developed software, the movies are analyzed to track all individual particles. Identifying particles with a minimum contrast with respect to the local background, particle tracking is performed using a nearest neighbor algorithm [16] (see Appendix C).

Two different sizes of "dragon green" (excitation $480 \mathrm{~nm}$, emission $520 \mathrm{~nm}$ ) fluorescent polymer nanospheres (Bangs Laboratories, Fishers, USA) were studied. First, a water dispersion of $0.19-\mu \mathrm{m}$ particles was diluted to a factor of 1888, 2382, 3368, 5826, and 14753 times. SPT experiments were carried out on each dilution and the concentrations were estimated using the single-particle tracking data. The theoretical number concentration in particles $/ \mathrm{ml}$ of the stock solution can be estimated using

$$
c_{\text {theoretical }}=\frac{6 \times 10^{10} \times S \rho_{L}}{\pi \rho_{S} d^{3}},
$$

where $S=1$ is the weight percentage of solids, with a relative standard deviation of $5 \%, \rho_{L}=1.00 \mathrm{~g} / \mathrm{cm}^{3}$ is the density of the suspension, $\rho_{S}=1.05 \mathrm{~g} / \mathrm{cm}^{3}$ is the density of the solid particles (all values according to manufacturer), and using dynamic light scattering the mean diameter of the particles was found to be $d=0.207 \mu \mathrm{m}$ with a standard deviation of $0.008 \mu \mathrm{m}$ (in correspondence with manufacturer results for that particular batch of nanospheres). Second, a water dispersion of $0.52-\mu \mathrm{m}$ particles was diluted to a factor of 142 , 190, 237, 380, and 750 times. Using dynamic light scattering once again, the mean diameter of the particles was found to be $d=0.497 \mu \mathrm{m}$ with a standard deviation of $0.009 \mu \mathrm{m}$ (in correspondence with manufacturer results for that particular batch of nanospheres). Using the standard propagation of error equation, theoretical number concentrations with standard deviations can be found for all dilutions, and compared with the SPT results.

Motion during image acquisition has an effect on the observed trajectory of a diffusing particle, which will affect the estimate of the diffusion coefficient, as discussed by others [20]. However, we consider here particles that are diffusing quickly with short acquisition times, so this effect is negligible. When performing experiments on the $0.52-\mu \mathrm{m}$ beads, the observation time was $5 \mathrm{~ms}$ and the position error approximately $2 \mathrm{~nm}$ (5500 photons collected). When performing experiments on the $0.19-\mu \mathrm{m}$ beads, the observation time was $2 \mathrm{~ms}$ and the position error approximately $6 \mathrm{~nm}$ (700 photons collected). The effect is thus small and this justifies the Gaussian approx-

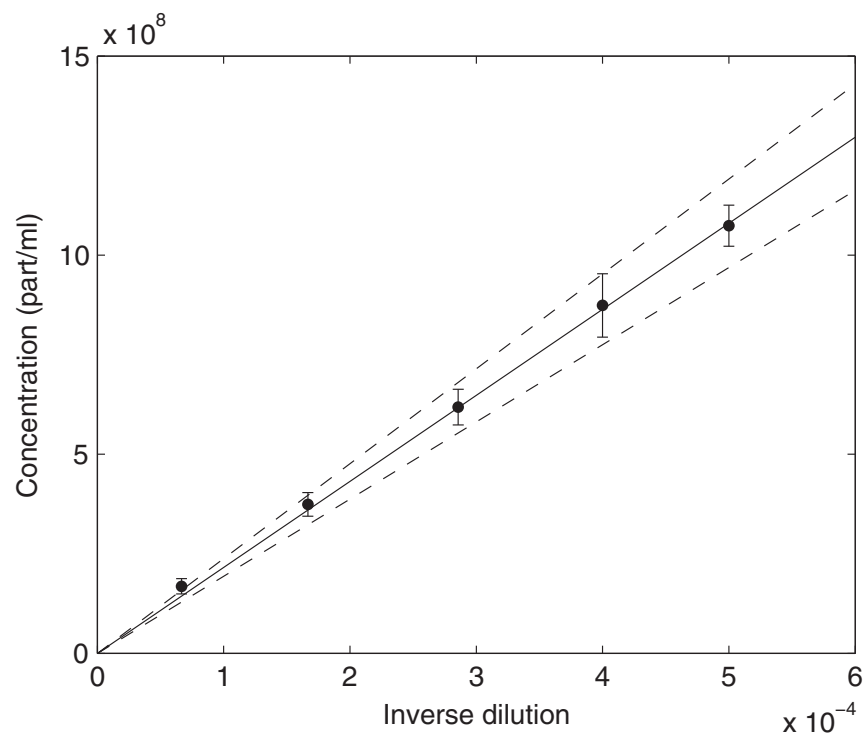

FIG. 3. Estimated concentrations for different dilutions of $0.19-\mu \mathrm{m}$ particles with estimated $95 \%$ confidence intervals. The concentration as estimated from the stock solution concentration (solid line) with estimated 95-\% confidence intervals (dashed lines) is shown. 


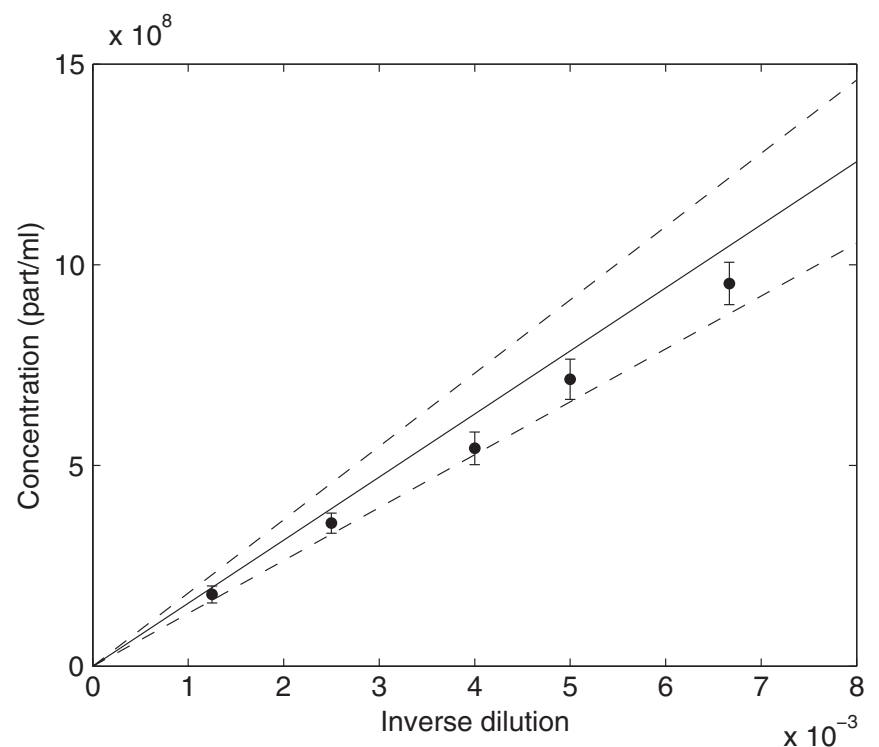

FIG. 4. Estimated concentrations for different dilutions of $0.52-\mu \mathrm{m}$ particles with estimated $95 \%$ confidence intervals. The concentration as estimated from the stock solution concentration (solid line) with estimated 95\% confidence intervals (dashed lines) is shown.

imation for the increments in the computations. We refer the reader to Appendix $\mathrm{C}$ for further details on the data acquisition.

As is clear from the results in Figs. 3 and 4, an excellent agreement was found between the theoretically and experimentally obtained concentration values. We would like to stress the fact that this result is obtained without any precalibration of the detection region volume.

Indeed, using the proposed model we estimate the detection region volume and number concentration simultaneously. To demonstrate that the detection region volume is not fixed but a variable parameter depending on the image processing settings, we have analyzed a single set of SPT movies of the

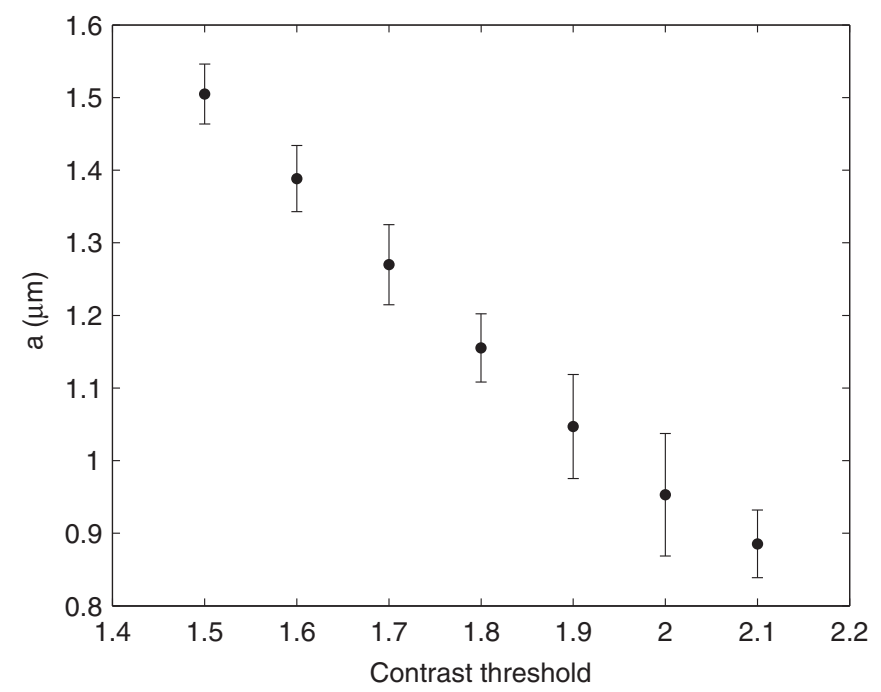

FIG. 5. Estimated values of $a$ with different contrast thresholds for the $0.52-\mu \mathrm{m}$ nanospheres, with estimated $95 \%$ confidence intervals.

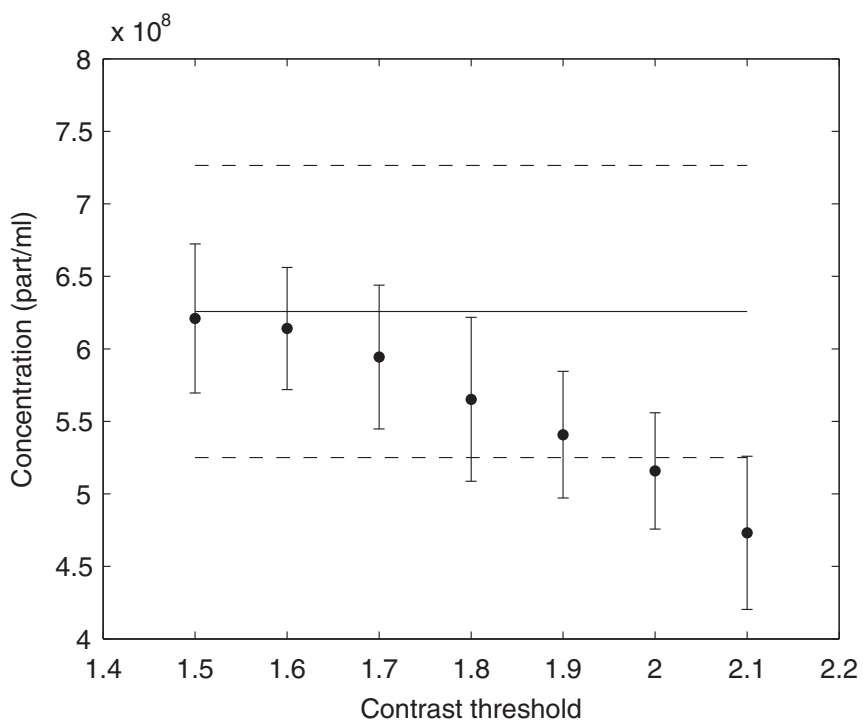

FIG. 6. Estimated values of concentration with different contrast thresholds for the $0.52-\mu \mathrm{m}$ nanospheres, with estimated $95 \%$ confidence intervals. The solid and dashed lines show the concentration as estimated from the stock solution concentration, and estimated $95 \%$ confidence intervals.

$0.52-\mu \mathrm{m}$ particles with different minimum contrast thresholds (see Appendix C), ranging from $C_{\min }=1.5$ to $C_{\min }=2.1$, in increments of 0.1 . For each of these settings the tracking depth parameter $a$ and the concentration were estimated; see Figs. 5 and 6, respectively. Note that as the contrast threshold increases, the estimated tracking depth decreases, which is natural considering that the particles furthest away from the focal plane have the least contrast and are removed.

Thanks to the inherent calibration of the detection region volume, the measured particle concentration varied considerably less than the tracking depth, which changed substantially in this range. The systematic decrease of the concentration that was in fact observed, however, may be explained in two ways. First, not all particles have exactly the same fluorescence intensity, so the dimmest ones get gradually and systematically excluded from the analysis when the contrast threshold is increased. Second, since the tracking depth decreases, the positive bias of the concentration estimates also decreases, when the contrast threshold is increased. While the former would indicate that a low threshold is preferable, the latter would indicate that a high threshold is preferable. To summarize, all SPT estimates (except for the $C_{\min }=2.0$ and 2.1 cases in Fig. 6) are within the $95 \%$ confidence interval for the theoretical concentration.

\section{DISCUSSION AND CONCLUSION}

We have derived and validated a theoretical model that makes use of the basic information in an SPT experiment to estimate the volume of the detection region. By estimating the axial size (the tracking depth) of the detection region, which is not directly observable, the volume of the $3 \mathrm{D}$ detection region can be estimated. This is performed by modeling of the trajectory length distribution of particles as a function of the axial size. The computations were simplified by 
assuming that the particles enter and exit the detection region only by means of axial diffusion, and the simulation study demonstrates that the bias is small under this approximation. Confidence intervals for both the tracking depth parameter and the concentration, giving accurate accounts of the uncertainty of the estimates, can also be acquired.

The method was experimentally validated by estimating the number concentration of different dilutions of 0.19 - and $0.52-\mu \mathrm{m}$ fluorescent carboxylated polymer nanospheres, and excellent agreement was found between the theoretically and experimentally obtained concentration values. It was also demonstrated that the results were robust with respect to changes in the minimum contrast threshold parameter.

In conclusion, we have demonstrated the validity of a method for accurate and absolute particle number concentration measurements, which are quite independent of variable factors like particle brightness and image processing settings. Considering the practical implications of avoiding precalibration measurements, this considerably increases the value and applicability of SPT as a tool for characterizing nanoparticle dispersions.

\section{ACKNOWLEDGMENTS}

The authors are indebted to Hugo Strand for ideas regarding the approximation by Gaussian basis functions. Acknowledgments go to Aila Särkkä for careful reading of the manuscript. Some of the computations were performed on C3SE (Chalmers Centre for Computational Science and Engineering) resources. This work has been carried out with financial support from the Swedish Foundation for Strategic Research (SSF) through Gothenburg Mathematical Modelling Center (GMMC), from the Swedish Research Council through Gothenburg Stochastic Centre, and from the Funds for Scientific Research, Flanders (Project No. G.0197.11). Hendrik Deschout acknowledges financial support from the Institute for the Promotion of Innovation through Science and Technology in Flanders (IWT), Belgium.

\section{APPENDIX A: INITIAL DISTRIBUTION IN THE DETECTION REGION}

Particles outside the detection region are uniformly distributed over $[-A,-a] \cup[a, A]$. This distribution has density function

$$
u(z)= \begin{cases}\frac{1}{2(A-a)}, & z \in[-A,-a] \cup[a, A], \\ 0, & \text { otherwise. }\end{cases}
$$

All particles then perform a random diffusive displacement by adding to their position a normally distributed increment with probability density

$$
G(z)=\frac{1}{\sqrt{2 D \Delta t}} \phi\left(\frac{z}{\sqrt{2 D \Delta t}}\right) .
$$

The resulting position is the sum of the two corresponding random variables with a probability density that is the convolution of their respective densities,

$$
h(z)=\int_{-\infty}^{\infty} u\left(z_{0}\right) G\left(z-z_{0}\right) d z_{0}
$$

which becomes

$$
h(z)=\frac{1}{2(A-a) \sqrt{2 D \Delta t}}\left(\int_{-A}^{-a}+\int_{a}^{A}\right) \phi\left(\frac{z-z_{0}}{\sqrt{2 D \Delta t}}\right) d z_{0} .
$$

Straightforward calculations yield that the non-normalized density of particles just having entered the detection region is

$$
\begin{aligned}
h(z)= & \frac{1}{2(A-a)}\left[\Phi\left(\frac{z+A}{\sqrt{2 D \Delta t}}\right)-\Phi\left(\frac{z+a}{\sqrt{2 D \Delta t}}\right)\right. \\
& \left.+\Phi\left(\frac{z-a}{\sqrt{2 D \Delta t}}\right)-\Phi\left(\frac{z-A}{\sqrt{2 D \Delta t}}\right)\right] .
\end{aligned}
$$

\section{APPENDIX B: NUMERICAL CONVOLUTION SCHEME}

We describe in detail the numerical convolution scheme. Computing $f_{k}$ for $k \geqslant 1$ can not be done analytically, so we expand the densities in a space of $n$ equidistant translates $\left\{\psi_{1}, \ldots, \psi_{n}\right\}$ of a Gaussian kernel, where

$$
\psi_{i}(z)=\frac{1}{w} \phi\left(\frac{z-m_{i}}{w}\right) .
$$

Here, $w$ is the width (standard deviation) of the kernels and $m_{i}$ is the center of the $i$ th kernel. Further we take $m_{i}-m_{i-1}=$ $w, i=2, \ldots, n, m_{1}=-a$ and $m_{n}=a$, yielding $w=2 a /(n-$ $1)$. In this way, the probability density is approximated inside of $[-a, a]$. More precisely, the approximation of the density $f_{k}$ is

$$
\tilde{f}_{k}(z)=\sum_{j} c_{j}^{(k)} \psi_{j}(z)
$$

where the weights $c_{j}^{(k)}$ are selected by exact interpolation, i.e., by demanding that the approximation be exact at the points $z=m_{i}, i=1, \ldots, n$. For $k=1$ we obtain $\tilde{f}_{1}\left(m_{i}\right)=f_{1}\left(m_{i}\right)$, $i=1, \ldots, n$, yielding the linear system

$$
U \mathbf{c}^{(1)}=f_{1}(\mathbf{m}),
$$

where $\quad \mathbf{c}^{(\mathbf{1})}=\left(c_{1}^{(1)}, \ldots, c_{n}^{(1)}\right)^{T}, \quad f_{1}(\mathbf{m})=\left(f_{1}\left(m_{1}\right), \ldots\right.$, $\left.f_{1}\left(m_{n}\right)\right)^{T}$, and

$$
U=\left(\begin{array}{llc}
\psi_{1}\left(m_{1}\right) & \cdots & \psi_{n}\left(m_{1}\right) \\
\vdots & \ddots & \vdots \\
\psi_{1}\left(m_{n}\right) & \cdots & \psi_{n}\left(m_{n}\right)
\end{array}\right)
$$

It is guaranteed that Eq. (B3) has a unique solution $\mathbf{c}^{(1)}=$ $U^{-1} f_{1}(\mathbf{m})$ since $U$ is invertible (and even positive definite). We refer the reader to [15] for further details on exact interpolation by radial basis functions. In every time step, the diffusive motion of particles is represented by convolving the current particle density with the Gaussian propagator Eq. (3). We illustrate the procedure and demonstrate the computation of $\tilde{f}_{k}$ from $\tilde{f}_{k-1}$. We put

$$
f_{k}^{\star}(z)=\tilde{f}_{k-1} * G(z)=\int_{-\infty}^{\infty} \tilde{f}_{k-1}\left(z_{0}\right) G\left(z-z_{0}\right) d z_{0},
$$

where we provisionally denote by $f_{k}^{\star}(z)$ a (nontruncated) density, which is not yet approximated in the Gaussian kernel 

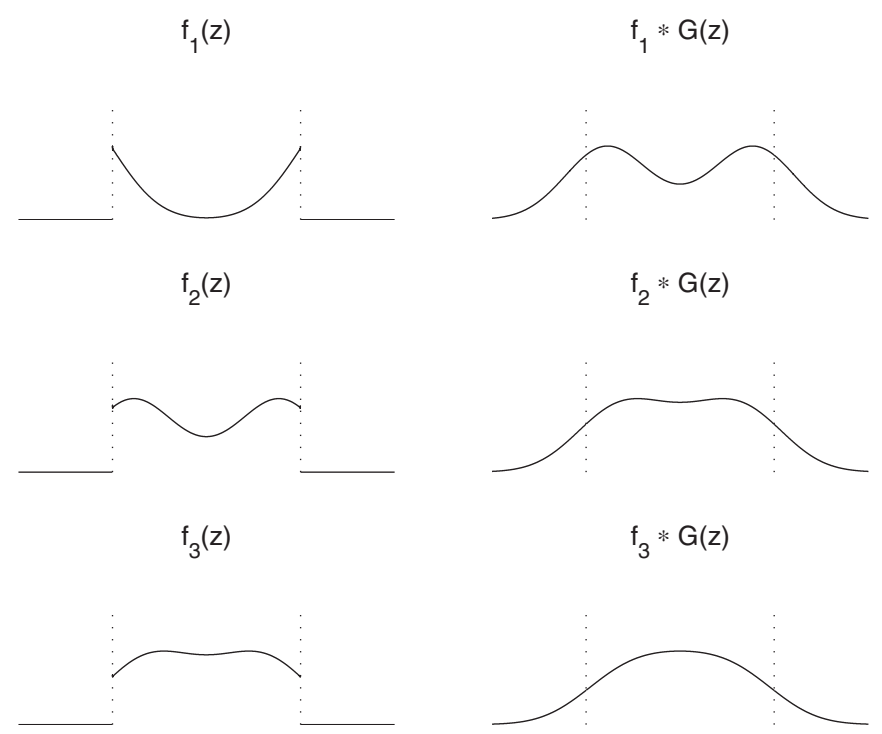

FIG. 7. Illustration of the procedure for computing the trajectory length distribution. $f_{1}(z)$ is the probability density of a particle that has just entered the detection region according to Eq. (1). Truncation outside of $[-a, a]$ of the convolution $f_{1} * G(z)$ yields a non-normalized density $f_{2}(z)$ which integrates to the probability that the particle still remains in the detection region for a second sampling time point, and so forth.

basis $\left\{\psi_{1}, \ldots, \psi_{n}\right\}$. Since the convolution of two Gaussians is Gaussian and because of linearity of convolution, we get

$$
f_{k}^{\star}(z)=\sum_{j} c_{j}^{(k-1)} \frac{1}{\sqrt{w^{2}+2 D \Delta t}} \phi\left(\frac{z-m_{j}}{\sqrt{w^{2}+2 D \Delta t}}\right),
$$

and in order to approximate this function within $[-a, a]$, we once again demand that the approximation be exact at the points $z=m_{i}$, i.e.,

$$
\begin{aligned}
\sum_{j} c_{j}^{(k)} \psi_{j}\left(m_{i}\right)= & \cdots \sum_{j} c_{j}^{(k-1)} \frac{1}{\sqrt{w^{2}+2 D \Delta t}} \\
& \times \phi\left(\frac{m_{i}-m_{j}}{\sqrt{w^{2}+2 D \Delta t}}\right), \quad i=1, \ldots, n,
\end{aligned}
$$

where $\mathbf{c}^{(k)}$ are the weights of the Gaussian kernels at the $k$ th time step. This leads to a linear system with the same matrix $U$ as above. Since the convolution at the points $m_{i}$ can be written as $V \mathbf{c}^{(k-1)}$, where

$$
\begin{aligned}
V= & \frac{1}{\sqrt{w^{2}+2 D \Delta t}} \\
& \times\left(\begin{array}{ccc}
\phi(0) & \cdots & \phi\left(\frac{(n-1) w}{\sqrt{w^{2}+2 D \Delta t}}\right) \\
\vdots & \ddots & \vdots \\
\phi\left(\frac{(n-1) w}{\sqrt{w^{2}+2 D \Delta t}}\right) & \cdots & \phi(0)
\end{array}\right),
\end{aligned}
$$

the entire step of convolution and approximation in the Gaussian basis can be written as

$$
U \mathbf{c}^{(k)}=V \mathbf{c}^{(k-1)},
$$
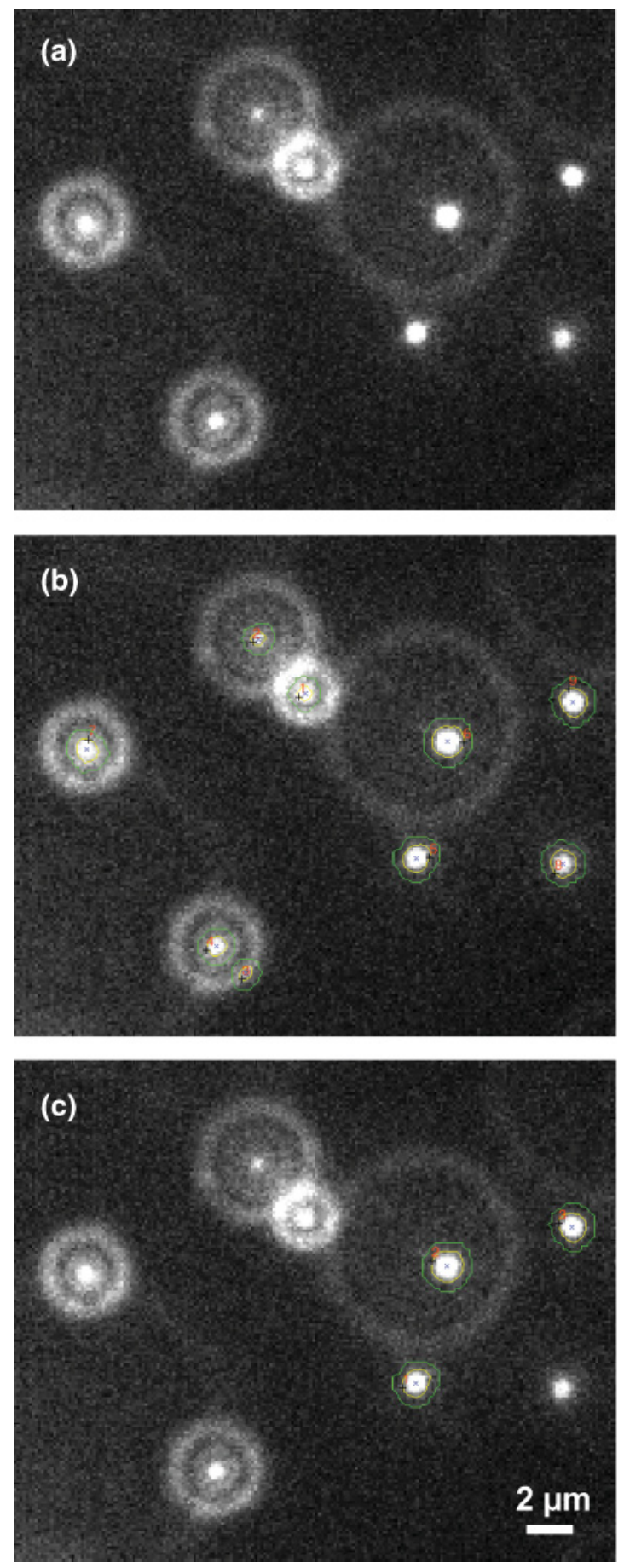

FIG. 8. (Color online) Result of different values for the minimum contrast to the local background. (a) Original image. (b),(c) Yellow (inner) contours indicate the particles that have been detected by the image processing software with a contrast to the local background larger than the minimum value. Green (outer) contours show the local background around each particle contour. The minimum contrast value is $C_{\min }=1.1 \mathrm{in}(\mathrm{b})$ and $C_{\min }=1.5 \mathrm{in}(\mathrm{c})$, resulting in nine and three detected particles, respectively.

which gives

$$
\mathbf{c}^{(k)}=U^{-1} V \mathbf{c}^{(k-1)} .
$$

The approximation of $f_{k}$ by $\tilde{f_{k}}$ can be made arbitrarily accurate by increasing $n$. For given $n$, it is sufficient to compute 
the matrix $U^{-1} V$ once to describe the diffusive dynamics and hence the change of the weights in every time step. These computations yield the distribution of $K$. Practically, to compute the cumulative distribution of $K$ we use

$$
P(K \leqslant k)=1-\int_{-a}^{a} f_{k+1}(z) d z \approx \cdots 1-\sum_{i} c_{i}^{(k+1)},
$$

using Eq. (5). This yields the distribution

$$
\begin{aligned}
P(K=k) & =P(K \leqslant k)-P(K \leqslant k-1) \\
& \approx \sum_{i}\left(c_{i}^{(k)}-c_{i}^{(k+1)}\right),
\end{aligned}
$$

which is implicitly a function of, most importantly, $a$ and $D$. The procedure is illustrated graphically in Fig.7.

\section{APPENDIX C: MATERIALS AND METHODS}

The SPT experiments were carried out on a custom-built laser wide-field epifluorescence microscope setup. A 100-mW Calypso 491-nm solid state laser (Cobolt, Solna, Sweden) was used for illumination. The laser beam passes through an acousto-optic tunable filter (AOTF; AA Optoelectronic, Orsay, France), which allows control of the intensity. The AOTF is synchronized with the charge-coupled device (CCD) camera in order to illuminate the sample only during the light integrating phase and to reduce photobleaching. The laser beam is directed through a $10^{\circ}$ light shaping diffuser (Physical Optics Corporation, Torrance, CA, USA), which in combination with an achromat lens in front of the microscope entrance provides wide-field Kohler illumination at the sample. The microscope is a Nikon TE2000E (Nikon BeLux, Brussels, Belgium) with a Nikon Plan Apochromat $60 \times$ NA1.20 water immersion objective lens. The fluorescence light coming from the sample is collected again by the objective lens and sent through the side port of the microscope toward the CCD camera. The fluorescence light is separated from the laser excitation light using a dichroic mirror and accompanying laser notch filter (AHF Analysentechnik, Tübingen, Germany). A pair of achromat lenses was placed between the CCD camera and the microscope side exit for an extra $1.5 \times$ magnification of the final image on the CCD chip. Since fast and sensitive image capture is required for SPT, an electron multiplying CCD camera was used (Cascade II:512; Roper Scientific, Tucson, AZ, USA). Image acquisition was performed using the Nikon Elements R imaging software. High-speed movies are recorded showing individual particles diffusing in the suspension. Typically $5 \mu \mathrm{l}$ of a water-particle solution was applied between a microscope slide and a cover glass with a double-sided adhesive sticker of $120 \mu \mathrm{m}$ thickness (corresponding to $A=60 \mu \mathrm{m}$ ) in between (Secure-Seal Spacer; Molecular Probes, Leiden, The Netherlands). This provides for a 3D environment in which the particles can diffuse freely, while the sample is sufficiently thin to avoid drift from convection. The microscope was always focused at least $20 \mu \mathrm{m}$ from the cover glass to avoid deviations from free diffusion due to the presence of the cover slip. All SPT experiments were carried out at $21^{\circ} \mathrm{C}$ and the full CCD chip was used, resulting in $512 \times 512$ pixel images. For the $0.19-\mu \mathrm{m}$ particles, the physical pixel size and the sampling interval were $0.1329 \mu \mathrm{m}$ and $38.2 \mathrm{~ms}$, respectively. For the $0.52-\mu \mathrm{m}$ particles, the physical pixel size and the sampling interval were $0.1329 \mu \mathrm{m}$ and $58.0 \mathrm{~ms}$, respectively. Forty videos for each dilution were acquired, all approximately $10 \mathrm{~s}$ long, with $k_{\min }=3$.

The experimentally obtained SPT movies are analyzed, using custom developed software, to track individual particles [16]. The first step to obtain the particle trajectories is to identify the particles in all frames of the SPT movie and to calculate their center location. The method for particle detection basically involves three steps. First, the nonuniform background is removed as much as possible. This can be done using a so-called unsharp filter, resulting in a filtered image where the particles stand out with good contrast against a much more uniform background. A first selection of possible objects can now be made by applying intensity thresholding to the filtered image, resulting in a binary image. Next, contours of individual objects in this binary image can be determined, and objects in the original image are supposed to lie within such contours. This allows us to calculate the object properties, such as the mean intensity and the center location. A second contour defining the local background is also calculated around each particle contour, which allows calculation of the mean local background intensity. Finally, having calculated all these properties, a last selection of particles can be made based on different criteria, such as minimum contour length, maximum contour surface, minimum contrast $C_{\min }$ with respect to the local background. The contrast $C$ is defined as the ratio of the mean intensity $I$ inside the contour and the local background intensity $B$,

$$
C=\frac{I}{B} \geqslant C_{\min }
$$

The higher the value of $C_{\min }$ the more particles are excluded, as illustrated in Fig. 8. Note that with increasing $C_{\min }$, more particles that are further away from the focal plane (typically dimmer and larger spots, sometimes with diffraction rings around them) are excluded, resulting in a smaller detection volume. Having found the particles with the right properties in all frames of an SPT movie, individual trajectories can be constructed by pairing corresponding particle center positions between images. A nearest neighbor algorithm is typically used to construct the trajectories.
[1] K. Remaut, N. Sanders, B. de Geest, K. Braeckmans, J. Demeester, and S. de Smedt, Mater. Sci. Eng., R 58, 117 (2007).

[2] S. Nune, P. Gunda, P. Thallapally, Y.-Y. Lin, M. Forrest, and C. Berkland, Expert Opin. Drug Deliv. 6, 1175 (2009).

[3] G. Chironi, C. Boulanger, A. Simon, F. Dignat-George, J.-M. Freyssinet, and A. Tedgui, Cell Tissue Res. 335, 143 (2009).
[4] L. Doevre, L. Plawinski, F. Toti, and E. Anglés-Cano, J. Neurochem. 110, 457 (2009).

[5] S. Khare and N. Dokholyan, Curr. Protein Pept. Sci. 8, 573 (2007).

[6] A. Morris, M. Watzky, and R. Finke, Biochim. Biophys. Acta 1794, 375 (2009). 
[7] M. Gaumet, A. Vargas, R. Gurny, and F. Delie, Eur. J. Pharm. Biopharm. 69, 1 (2008).

[8] V. Vysotskii, O. Uryupina, A. Guselnikova, and V. Roldugin, Colloid J. 71, 739 (2009).

[9] E. van Gaal, G. Spierenburg, W. Hennink, D. Crommelin, and E. Mastrobattista, J. Controlled Release 141, 328 (2009).

[10] I. Montes-Burgos, D. Walczyk, P. Hole, J. Smith, I. Lynch, and K. Dawson, J. Nanopart. Res. 12, 47 (2010).

[11] K. Braeckmans, K. Buyens, W. Bouquet, C. Vervaet, P. Joye, F. de Vos, L. Plawinski, L. Doeuvre, E. Anglés-Cano, N. Sanders, J. Demeester, and S. de Smedt, Nano Lett. 10, 4435 (2010).

[12] K. Braeckmans, K. Buyens, B. Naeye, D. Vercauteren, H. Deschout, K. Raemdonck, K. Remaut, N. Sanders, J. Demeester, and S. de Smedt, J. Controlled Release 148, 69 (2010).

[13] S. Du, K. Kendall, S. Morris, and C. Sweet, J. Chem. Technol. Biotechnol. 85, 1223 (2010).
[14] H. C. Berg, Random Walks in Biology (Princeton University Press, Princeton, NJ, 1993).

[15] M. D. Buhmann, Radial Basis Functions: Theory and Implementations (Cambridge University Press, Cambridge, UK, 2003).

[16] K. Braeckmans, D. Vercauteren, J. Demeester, and S. de Smedt, in Nanoscopy and Multidimensional Optical Fluorescence Microscopy, edited by E. Diaspro (Chapman and Hall, London, 2010).

[17] K. Jaqaman, D. Loerke, M. Mettlen, H. Kuwata, S. Grinstein, S. Schmid, and G. Danuser, Nat. Meth. 5, 695 (2008).

[18] Y. Pawitan, In All Likelihood: Statistical Modelling and Inference Using Likelihood (Oxford University Press, Oxford, UK, 2001).

[19] B. Efron and R. Tibshirani, An Introduction to the Bootstrap (Chapman and Hall, London, 1993).

[20] X. Michalet, Phys. Rev. E 82, 041914 (2010). 